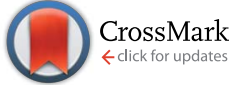

Cite this: RSC Adv., 2015, 5, 6033

Received 10th October 2014 Accepted 15th December 2014

DOI: $10.1039 / c 4 r a 12156 b$

www.rsc.org/advances

\section{Effective formulation and processing of nanofilled carbon fiber reinforced composites}

\author{
L. Guadagno, ${ }^{* a}$ M. Raimondo, ${ }^{a}$ U. Vietri, ${ }^{a}$ L. Vertuccio, ${ }^{a}$ G. Barra, ${ }^{a}$ B. De Vivo, ${ }^{b}$ \\ P. Lamberti, ${ }^{b}$ G. Spinelli, ${ }^{b}$ V. Tucci, ${ }^{b}$ R. Volponi, ${ }^{c}$ G. Cosentino ${ }^{c}$ and F. De Nicola ${ }^{c}$
}

This work describes a successful approach toward the development of a carbon fiber-reinforced composite based on an optimized nanofilled resin for industrial applications. The epoxy matrix is prepared by mixing a tetrafunctional epoxy precursor with a reactive diluent which allows reduction of the viscosity of the epoxy precursor and facilitation of the dispersion of $0.5 \%$ wt multiwall carbon nanotubes. The proper choice of the viscosity value and the infusion technique allow improvement of the electrical properties of the panels. The obtained in-plane electrical conductivity is about $20 \mathrm{kS} \mathrm{m}^{-1}$, whereas a value of $3.9 \mathrm{~S} \mathrm{~m}^{-1}$ is achieved for the out of plane value. Such results confirm that the fibers govern the conduction mechanisms in the direction parallel to the fibers, whereas the percolating path created by the effective distribution of carbon nanotubes achieved by resin formulation and adopted processing approach lead to a significant enhancement of the overall electrical performance of the composites.

\section{Introduction}

In recent years the use of carbon fiber-reinforced composites (CFRC) has continuously expanded, particularly in weightsensitive applications, such as aircraft and space vehicles. In particular, the increasing application of epoxy-based thermosetting composite materials in the aircraft industry was driven by the possibility to attain a significant weight reduction with respect to traditional metallic materials in the fabrication of structural parts. However, composites based on epoxy resins exhibit some rather inherent unsatisfactory characteristics, such as poor electrical conductivity. Epoxy resins are known, in fact, for their good or excellent properties covering an extensive range of applications, ${ }^{1-3}$ but at same time for their undesired electrical insulating behavior which limits their applicability as aerospace and aeronautical materials and, in general, where antistatic properties are required. One attempt to increase their application range is to incorporate nanoscale conductive fillers that are characterized by intrinsically high electrical conductivity. ${ }^{4-12}$ In order to choose an effective epoxy mixture, the intended application and consequently the properties required for the finished product have to be carefully considered. In the case of epoxy matrix it is known that the structure of the resin strongly governs its chemical and some of the physical

${ }^{a}$ Department of Industrial Engineering, University of Salerno, Via Giovanni Paolo II, 132-84084 Fisciano (SA), Italy. E-mail: lguadagno@unisa.it

${ }^{b}$ Department of Information Engineering, Electrical Engineering and Applied Mathematics, University of Salerno, Via Giovanni Paolo II, 132 - 84084 Fisciano (SA), Italy

${ }^{c}$ CIRA Italian Aerospace Research Centre, Advanced Materials and Technologies Lab, Via Maiorise, 81043 Capua (CE), Italy properties. The number of reactive sites in the epoxy precursors controls the functionality directly acting on the cross-linking density. This, combined with the nature of the hardener agent, the functionality, the stoichiometry and the curing cycle determines the finished properties of the cured resin especially in terms of mechanical and thermal properties. In order to obtain high mechanical and electrical performance, in this work, multi-wall carbon nanotubes (MWCNTs) were embedded inside an epoxy resin based on a mixture of tetraglycidylmethylenedianiline (TGMDA) and 1,4-butandioldiglycidylether (BDE). This particular epoxy formulation has proven to be very effective for improving nanofiller dispersion due to a decrease in the viscosity ${ }^{13-16}$ and, in addition, it has been found to reduce the moisture content which is a very critical characteristic for aeronautic materials. ${ }^{17}$ The chemical composition of this epoxy formulation reduces the sorption at equilibrium of liquid water $\left(C_{\mathrm{eq}}\right)$ of about $35 \%$. This percentage is very relevant for epoxy mixtures to be applied as structural materials in the aeronautics; in fact, absorbed moisture reduces the matrixdominated mechanical properties. Absorbed moisture also causes the material to swell. In addition, during freeze-thaw cycles, the absorbed moisture expands during freezing and can crack the material. The amount of MWCNTs inside the epoxy mixture used to impregnate plies of carbon fiber (CF) cloths was chosen by studying the electrical behavior of the nanofilled resin alone (without $\mathrm{CFs}$ ). The electrical percolation threshold (EPT), i.e. the value of filler content ensuring the transition from insulating to conducting behaviour of the composite, was found to be in the range $[0.1 ; 0.32] \%$ wt. Also the AC measurements confirmed that the EPT ranges between $[0.1 ; 0.32] \%$ wt. 
An amount of $0.5 \% \mathrm{wt}$, beyond the EPT was, then, adopted to prepare the nanofilled epoxy mixture used to manufacture carbon-fiber reinforced panels. An economic and efficient mean of producing high performance fiber-reinforced panels containing nanofilled resin which impregnates CFs is adopted which is especially useful when, as the considered case, the initial viscosity of epoxy precursors/mixture appears to be very high for an infusion process. This, indeed, is a non-trivial problem in the case of aeronautic epoxy mixtures containing a percentage of conductive nanofillers able to obtain samples in the nanofiller concentration range beyond the EPT.

The implemented technique to infuse the nano-filled resin into a carbon fiber dry preform allows to obtain remarkable electrical properties for the manufactured panels. A detailed morphological analysis is also presented allowing to explain the mechanisms which lead to the achievement of the very good values found for the electrical conductivity.

\section{Experimental}

\subsection{Materials}

2.1.1. Nanofilled resin. The epoxy matrix was prepared by mixing an epoxy precursor, tetraglycidylmethylenedianiline (TGMDA) (epoxy equivalent weight [117; 133] g per eq.), with an epoxy reactive monomer 1,4-butanedioldiglycidyl ether (BDE) that acts as a reactive diluent. The curing agent investigated for this study is 4,4-diaminodiphenyl sulfone (DDS). The epoxy mixture was obtained by mixing TGMDA with BDE monomer at a concentration of $80: 20 \%$ (by wt) epoxide to flexibilizer. The hardener agent was added at a stoichiometric concentration with respect to all the epoxy rings (TGMDA and BDE), this mixture will be named hereunder T20BD formulation. This epoxy formulation hardened with DDS has shown to be characterized by a good flame resistance with a limiting oxygen index of $27 \%$, even without addition of antiflame compounds. ${ }^{18}$

The MWCNTs (3100 Grade) were obtained from Nanocyl S.A. Transmission electron microscopy (TEM) investigation has shown for MWCNTs an outer diameter ranging from $10 \mathrm{~nm}$ to $30 \mathrm{~nm}$. The length of MWCNTs is from hundreds of nm to some micrometer. The number of walls varies from 4 to 20 in most nanotubes. The specific surface area of MWCNTs determined with the BET method is around $250-300 \mathrm{~m}^{2} \mathrm{~g}^{-1}$; the carbon purity is $>95 \%$ with a metal oxide impurity $<5 \%$ as it results by thermogravimetric analysis. Epoxy blend and DDS were mixed at $120{ }^{\circ} \mathrm{C}$ and the MWCNTs were added and incorporated into the matrix by using an ultrasonication for $20 \mathrm{~min}$. An ultrasonic device, Hielscher model UP200S (200 W, $24 \mathrm{kHz}$ ) was used. The epoxy mixture used to manufacture the panels was filled with $0.5 \%$ wt of MWCNTs. This nanofilled sample will be named hereunder T20BDCNTs formulations. This concentration was chosen because the curves of DC volume conductivity $v$. MWCNTs concentration highlight that the electrical percolation threshold (EPT) is lower than $0.32 \%$ wt, therefore for this amount of MWCNTs the nanofilled formulation is beyond the EPT. ${ }^{17,19}$ This formulation is also characterized by good dynamic mechanical properties. ${ }^{20}$
2.1.2. CFRCs - manufacturing process. CFRCs were manufactured by Resin Film Infusion (RFI) using a non-usual technique to infuse a nano-filled resin into a carbon fiber dry perform. ${ }^{21}$ This new technique allows to overcome drawbacks related to non optimal values of the viscosity for a RFI process and is therefore particularly advantageous for nanofilled resins where the presence of nanofillers increases the viscosity values hindering the injection of the nanofilled epoxy. A well-known process for the manufacturing of high performance resinbased composite materials is the resin film infusion. In this process, a dry carbon fiber preform is placed in a vacuum bag and the resin is injected from an edge of preform while in the other is vent the vacuum so the resin flow throw the length of the preform. The scheme can be seen in Fig. 1.

Optimum value of viscosity required for this process is lower than 0.3 $\mathrm{Pa}$ s. In literature, it is possible to find a theoretical maximum limit of $0.8 \mathrm{~Pa} \mathrm{s.}^{22}$ Often a nanofilled resin exceeds the limit of $0.8 \mathrm{~Pa} \mathrm{~s}$; then the usual liquid infusion becomes unfeasible. To overcome this critical point, a thin layer of liquid epoxy mixture containing MWCNTs ( $0.5 \% \mathrm{wt})$ was spread on a release films (Release Ease 234 TFP-HP Airtech); then a dry preform $(400 \mathrm{~mm} \times 400 \mathrm{~mm})$ made laminating 7 plies of carbon fiber cloths (SIGMATEX (UK) LDT 193GSM (grams square meter)/PW (plain wave)/HTA40 E13 3K (3000 fibers each tow)) was placed on mixture forcing it to flow throw the thickness of the preform using an external supplementary pressure inside an autoclave. In this way the length of the impregnation path is considerably reduced and the process can be forced by means of the pressure application. A further advantage of this technology is associated to the smaller length of the infiltration path which reduces the effects of infiltration through the preform, ensuring in this way a more uniform distribution of the nanofiller through the panel thickness. In fact, the edges of the preform were sealed to force the resin to flow only through the thickness (see Fig. 2A). The laminate was covered by a porous release film and a distribution media to allow to the resin to escape from the upper side and a breather media to receive the excesses of the resin (see Fig. 2B). Finally it was placed in a vacuum bag and the laminate was transferred into the autoclave (see Fig. 2C).

\subsection{Methods}

\subsubsection{Morphological investigation}

Nanofilled resin T20BDCNTs. Fracture surfaces of the composite specimens T20BDCNTs were investigated after etching procedure to remove a fraction of resin and better

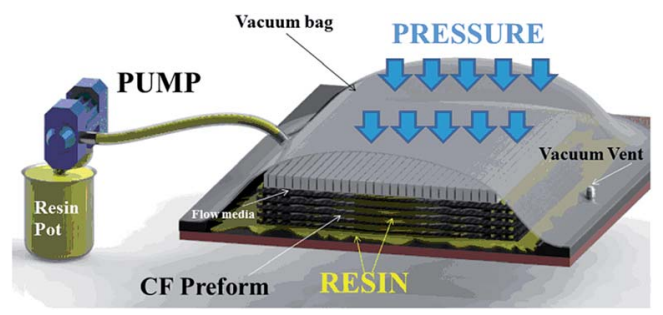

Fig. 1 Scheme of liquid resin infusion technique. 

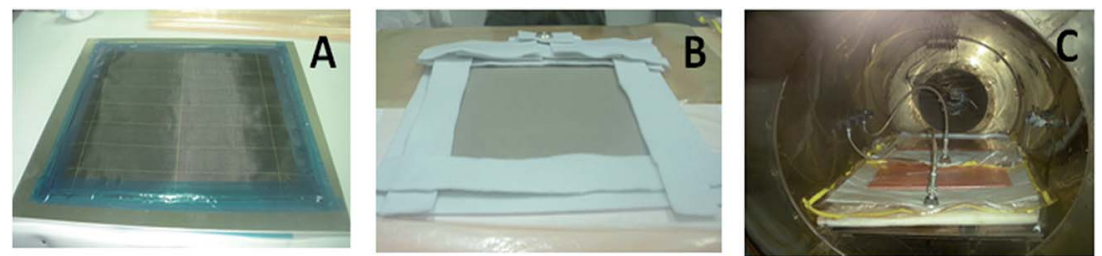

Fig. 2 Steps of laminate preparation.

evidence the morphological feature. Micrographs were captured with a LEO 1525 Scanning Electron Microscope (SEM).

Panels of CFRCs. Strips of CFRCs were cut out from the panels and analyzed in direction parallel (i.e. in plane) and perpendicular (i.e. out of plane) to the panel plane. Some of the samples were treated with a strong etching reagent and observed using conventional techniques of Field Emission Scanning Electron Microscope (FESEM, mod. LEO 1525, Carl Zeiss SMT AG, Oberkochen, Germany). The etching reagent was prepared by stirring $1.0 \mathrm{~g}$ potassium permanganate in a solution mixture of $95 \mathrm{ml}$ sulphuric acid (95-97\%) and $48 \mathrm{ml}$ orthophosphoric acid (85\%). The filled resins were immersed into the fresh etching reagent at room temperature and held under agitation for 36 hours. Subsequent washings were done using a cold mixture of 2 parts by volume of concentrated sulphuric acid and 7 parts of water. Afterwards the samples were washed again with $30 \%$ aqueous hydrogen peroxide to remove any manganese dioxide. The samples were finally washed with distilled water and kept under vacuum for 5 days at room temperature. The samples were placed on a carbon tab previously stuck to an aluminum stub (Agar Scientific, Stansted, UK). The samples were covered with a $250 \AA$-thick gold film using a sputter coater (Agarmod. $108 \mathrm{~A}$ ).

2.2.2. Panels of CFRCs - electrical measurements. Carbon fiber composites can be classified on the basis of the length (short or continuous) of the used fibers. Continuous carbon fibers, aligned unidirectionally or forming a woven fabric have stronger effect on the mechanical, electrical, and thermal properties and give rise to composites characterized by higher anisotropy than that of found with short fibers. ${ }^{23}$ Therefore, for the conductivity of the continuous CFRCs here analyzed, two types of measurements of the volumetric DC conductivity can be obtained: the in plane, $\sigma_{\mathrm{v} \|}$, and the out of plane, $\sigma_{\mathrm{v} \perp}$. The measurement of $\sigma_{\mathrm{v} \|}$ is performed by using a strip sample, named DST07, of about $1.7 \times 4.0 \times 0.17 \mathrm{~cm}^{3}$ whereas $\sigma_{\mathrm{v} \perp}$ is carried out on a square sample, named DLS01, of about $6 \times 6 \times 0.17 \mathrm{~cm}^{3}$.

Before performing the electrical measurements, the samples are cleaned with acetone and thermally pretreated at $80{ }^{\circ} \mathrm{C}$ for $24 \mathrm{~h}$. Then, contacts made with silver paint (Alpha Silver Coated Copper Compound Screening, with a thickness of about $50 \mu \mathrm{m}$ and a resistivity of $0.7 \Omega$ square) are deposited. In order to obtain the conductivity values, two multimeters (HP 34401A, HP 3408A) and an electrometer (Keithley 6514A) are used. A BINDER climatic chamber Model FP 53 has been used to analyze the temperature dependence of the conductivities in the range [30-90] ${ }^{\circ} \mathrm{C}$ with step $\Delta T=10^{\circ} \mathrm{C}$.

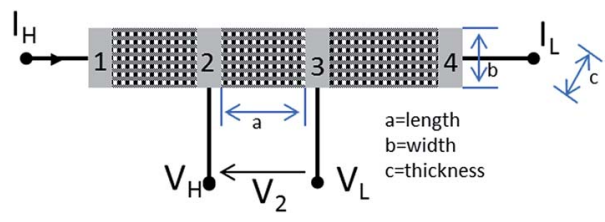

Fig. 3 Set-up for the measurement of the in plane volumetric DC conductivity.

The measurement of $\sigma_{\mathrm{v} \|}$, is performed by using the 4-probe method as schematically shown in Fig. 3.

In Table 1 the geometrical dimensions of the electrode configuration are reported together with the relative uncertainty in the geometric dimensions in the form $x \pm \Delta x$. In this case the conductivity of the material samples under test is calculated using the following expression:

$$
\sigma_{\mathrm{v} \|}=\frac{1}{R_{\mathrm{c}}} \frac{a}{b c}
$$

where $R_{\mathrm{c}}=V_{2} / I_{\mathrm{H}}$ is the measured resistance of the sample and $a$, $b$ and $c$ the geometric quantities of Fig. 3 .

The measurement of $\sigma_{\mathrm{v} \perp}$ is performed by using the 3-probe method with the configuration given in Fig. 4 . This technique is adopted in order to avoid any superficial leakage currents.

In Table 2 the geometrical dimensions of the electrode configuration for the square case DLS01 are reported together with the relative uncertainty in the geometric dimensions in the form $x \pm \Delta x$.

In Fig. $4, D_{1}$ and $D_{2}$ are the diameter of the inner and outer electrode of the top side, respectively, $D_{3}$ is the electrode diameter of the bottom side and $s$ is the thickness of the CFRC sample.

By setting $g$ and $K_{\mathrm{v}}$ equal to:

$$
g=\frac{D_{2}-D_{1}}{2} ; K_{\mathrm{v}}=\left(\frac{D_{1}}{2}+0.5 \frac{g}{2}\right)^{2}
$$

the out of plane conductivity of the CFRC is given by:

$$
\sigma_{\mathrm{v} \perp}=\frac{1}{R_{\mathrm{c}}} \frac{s}{\pi K_{\mathrm{v}}}
$$

where $R_{\mathrm{c}}=V_{\mathrm{m}} / I_{1}$ is the measured resistance of the sample.

2.2.3. Determination of the volumetric fraction of carbon fiber. The volumetric fraction of carbon fiber is a fundamental value to evaluate the final proprieties in a carbon fiber article. In aeronautic application the mechanical performance of a 
Table 1 Geometrical dimension of the electrode configuration for the strip sample

\begin{tabular}{lllllll}
\hline Sample & $a[\mathrm{~cm}]$ & $\Delta a[\mathrm{~cm}]$ & $b[\mathrm{~cm}]$ & $\Delta b[\mathrm{~cm}]$ & $c[\mathrm{~mm}]$ & $\Delta c[\mathrm{~mm}]$ \\
\hline Sample DST07 & 1.11 & 0.02 & 1.61 & 0.02 & 1.31 & 0.01
\end{tabular}

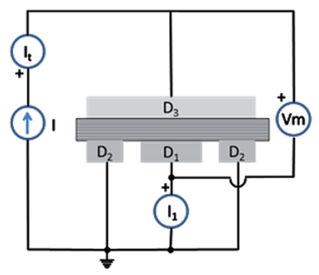

a
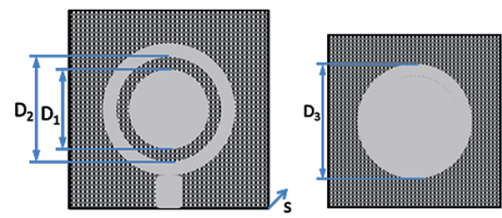

b
Fig. 4 Arrangement for the measurement of the out of plane volumetric DC conductivity: electric circuit (a); geometric structure of the top and down electrodes (b).

composite is maximized at optimal values of volumetric fraction of carbon fiber very close to $60 \%$.

The volume fraction was calculated using the following formula:

$$
V_{\mathrm{f}}=\frac{n \times \mathrm{gsm} \times 10^{-4}}{s \rho}
$$

in which $n$ is the number of plies composite configuration, gsm is the actual weight of carbon fabric before the impregnation (grams per square meter), $s$ is the thickness $(\mathrm{cm})$ of composite, $\rho$ is the density of the fiber (IM7), $\rho=1.78 \mathrm{~g} \mathrm{~cm}^{-3}$.

2.2.4. Rheology. TA Instruments AR 2000 dual head Rheometer was used to analyze the viscoelastic behavior in oscillation tests. Parallel plate $\alpha=40 \mathrm{~mm}$ was selected as appropriate geometry and the gap was set at a value of $300 \mu \mathrm{m}$. Strain sweep were carried out at constant frequency of $1 \mathrm{~Hz}$, in order to find the linear viscoelastic region. Temperature Sweep, varying the temperature from $[60 ; 120]^{\circ} \mathrm{C}$ a rate of $3{ }^{\circ} \mathrm{C} \min ^{-1}$, was performed at the frequency of $1 \mathrm{~Hz}$ and the strain $\%$ of $5 \%$, determined from the strain sweeps, within the linear viscoelastic region.

\section{Results and discussion}

\subsection{Nanofilled epoxy mixture}

3.1.1. Morphological investigation. In order to analyze the homogeneity of the nanofiller dispersion in the polymeric matrix, the sample with MWCNTs at $0.32 \%$ wt was investigated by means of scanning electron microscopy (see Fig. 5). A careful observation evidences a good level of MWCNTs

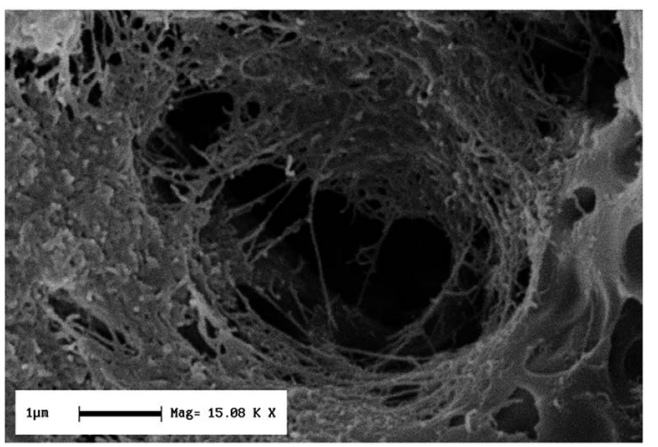

Fig. 5 SEM image of the fracture surface of the composite with $0.32 \%$ wt loading of MWCNTs.

interconnections able to form conductive paths also at the low nanofiller percentage of $0.32 \% \mathrm{wt}$. This assertion is confirmed by the data related to the electrical conductivity of the nanofilled samples.

3.1.2. Electrical behavior. In a previous paper ${ }^{19}$ the authors have shown that for the proposed epoxy mixture at different filler loading $\phi$, the DC conductivity $v s$. the filler concentration shows the typical behaviour predicted by the percolation theory: ${ }^{12,19}$

$$
\sigma=\sigma_{0}\left(\phi-\phi_{\mathrm{c}}\right)^{t}
$$

where $\phi_{\mathrm{c}}$ is the electrical percolation threshold (EPT), $\sigma_{0}$ is the theoretical conductivity at high filler concentrations and $t$ is an exponent linked to the structural dimensionality of the filler distribution inside the matrix. In particular, the measured data have been used to extract $\nu_{\mathrm{c}}=100 \times \phi_{\mathrm{c}}=0.22 \%$, that is the minimum weight percentage wt $\%$ ensuring that the percolation regime has been achieved within the nanofilled resin. This means that the electrical behaviour of the nanofilled epoxy mixture changes from that of an insulating material, similar to that of the T20BD matrix hosting the filler, to that of a conducting material whose electromagnetic behaviour is governed by the paths formed by the MWCNTs inside the matrix. From the same data also an estimation of the morphological parameter is obtained, whose high value $(t=2.2)$ indicates that the MWCNTs, as also confirmed by the morphological investigations illustrated by the SEM image of Fig. 5, are organized in a three-dimensional network.

The volume DC conductivity of the nanofilled epoxy mixture is $\sigma_{\mathrm{v}}=0.142 \mathrm{~S} \mathrm{~m}^{-1}$ for the sample at a loading of $0.5 \mathrm{wt} \%$. The obtained value confirms that the mixture is above the EPT. Also the measurements of the electrical properties in the frequency range $[0.1 ; 1000] \mathrm{kHz}$, not shown here, confirm that the percolation threshold is in the range $[0.1 ; 0.32] \% \mathrm{wt}$ and that at $0.5 \%$

Table 2 Geometrical dimension of the square sample electrode configuration

\begin{tabular}{lllllll}
\hline Sample & $D_{1}[\mathrm{~cm}]$ & $\Delta D_{1}[\mathrm{~cm}]$ & $D_{2}[\mathrm{~cm}]$ & $\Delta D_{2}[\mathrm{~cm}]$ & $s[\mathrm{~mm}]$ & $\Delta s[\mathrm{~mm}]$ \\
\hline Sample DLS01 & 2.50 & 0.02 & 2.80 & 0.02 & 1.34 & 0.01
\end{tabular}


Table 3 Values of the viscosity $\eta^{*}[\mathrm{~Pa} \mathrm{~s}]$ at different temperatures for pristine resin and filled with $0.5 \%$ wt of MWCNT

\begin{tabular}{lll}
\hline & & $0.5 \% \mathrm{wt}$ \\
Temperature $\left[{ }^{\circ} \mathrm{C}\right]$ & Pristine & of MWCNTs \\
\hline 80 & 5.26 & 7.83 \\
90 & 2.11 & 5.82 \\
100 & 0.96 & 4.49 \\
110 & 0.52 & 3.58 \\
120 & 0.31 & 3.05
\end{tabular}

wt the composite performs as a conducting material. In fact, the AC conductivity is constant in the analysed frequency range and close to the DC value. Moreover, the measured real part of the relative permittivity is about two order of magnitude greater ( $\left.\varepsilon_{\mathrm{r}} \cong 1000\right)$ than the value obtained for the unfilled T20BD matrix, with a frequency dependence showing the dispersion typical of a loss material.

\subsection{Carbon fiber reinforced panels}

3.2.1. Morphological investigation. Table 3 shows the values of the viscosity $\eta^{*}$ of the unfilled and nanofilled formulation at different temperatures.

Data shown in Table 3 highlight that, although the viscosities of the unfilled and nanofilled resins have been strongly lowered with the mixing of the epoxy precursor with the reactive diluent, the usual liquid infusion process is not feasible. The technique described in the section "CFRCs - Manufacturing Process" allows to overcome these criticalities. Impregnation conditions (curing cycle and pressure) of CFRC panels is shown in Fig. 6.

An optical picture of the manufactured panels is shown in Fig. 7.

All the manufactured panels are characterized by a calculated volume fiber fraction $V_{\mathrm{f}}$ between 0.55 and 0.56 . Fig. $8 \mathrm{a}$ and $b$ show the SEM images at two different magnification of the some cross sectional areas of the manufactured panel (not etched sample). Fig. 8a also clearly shows the number of plies.

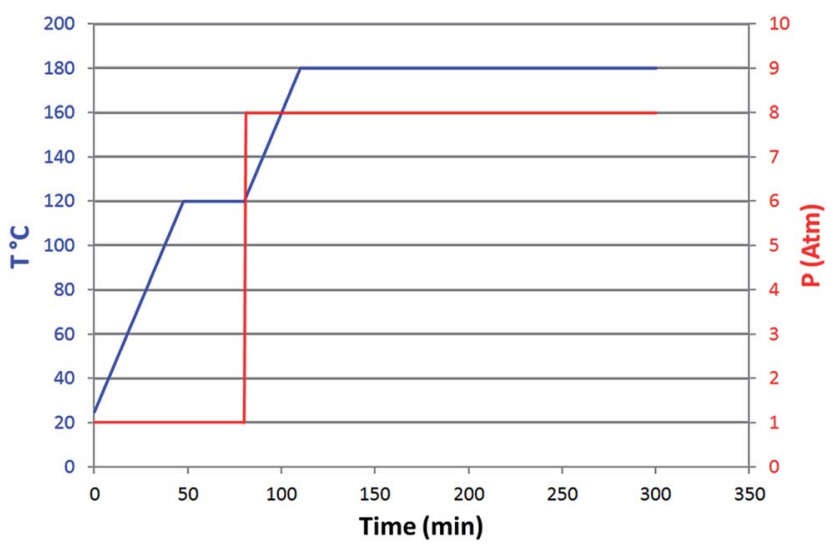

Fig. 6 Curing cycle and pressure for the Impregnation process.

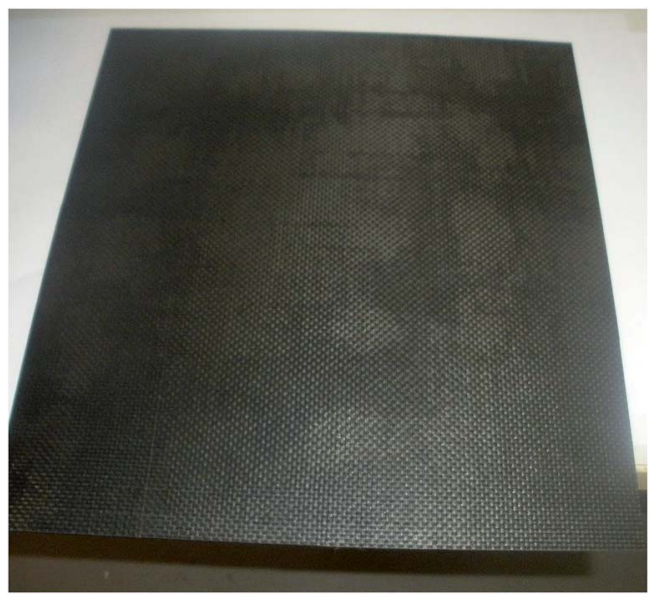

Fig. 7 Manufactured panel $(33 \mathrm{~cm} \times 30 \mathrm{~cm})$.

Because the investigated region is comprised between two nodes of the fabric mesh and relates to a sectional area (where the sample was punched out), we can deduce that a good interfacial bonding between carbon fiber fabric and epoxy resin has been achieved. FESEM image on the top side of the CFRC panel surface was acquired after a strong etching procedure and

\section{PLIES (BIDIRECTIONAL CARBON FIBERS FABRIC)}
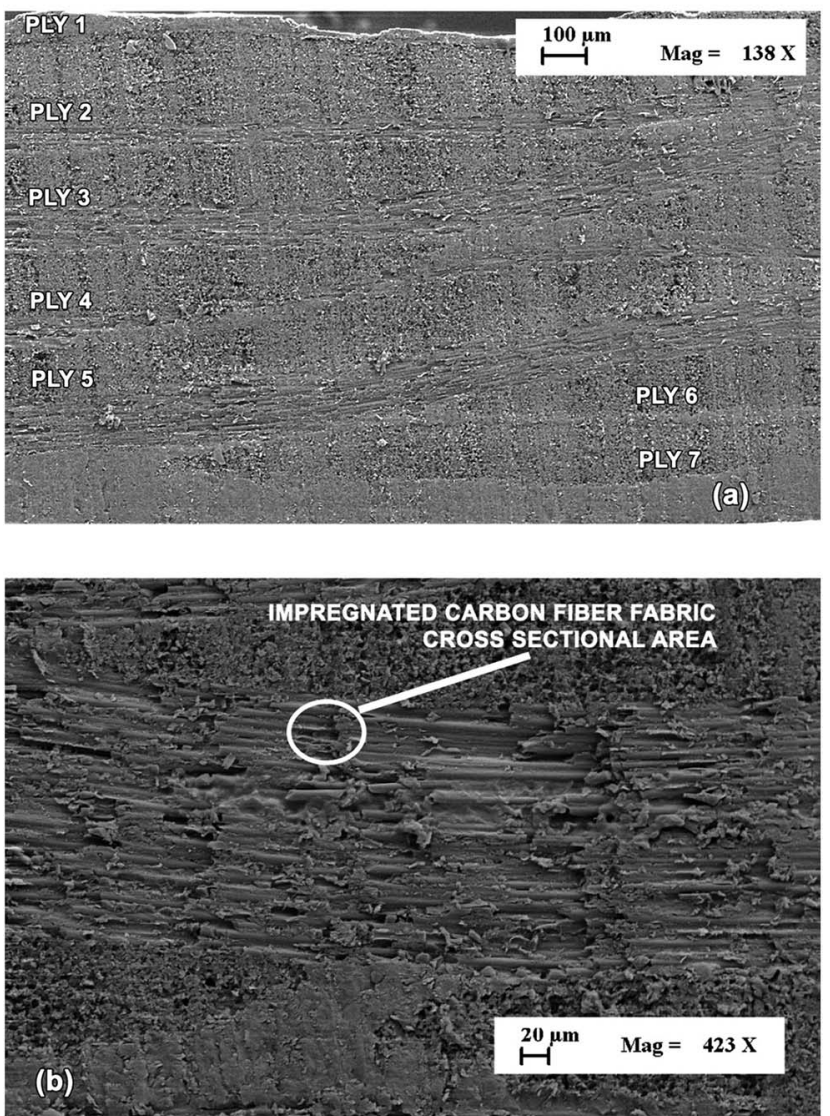

Fig. 8 SEM images, at two different magnification, of the cross sectional area of the manufactured panel. 

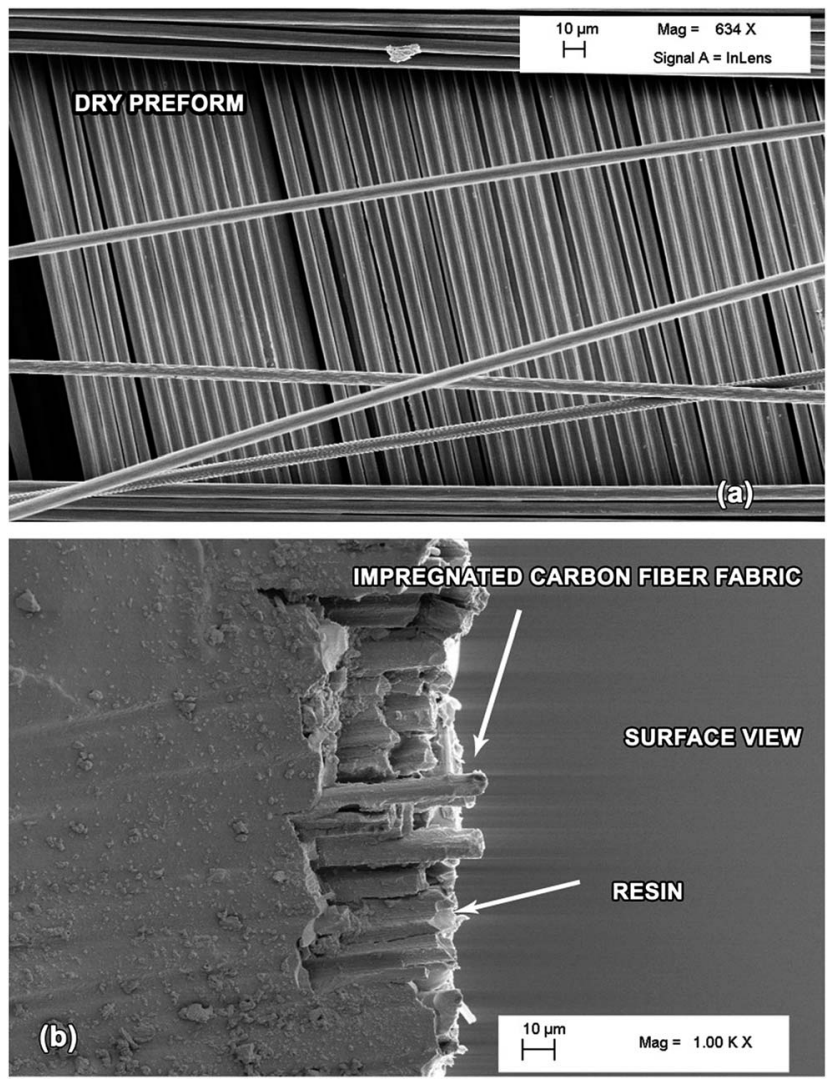

Fig. 9 FESEM image of the dry perform (a) and of the top side of the CFRC surface (b).

it is shown in Fig. 9. In this figure the dry preform fabric is also shown on the top to allow a comparison.

The image highlights that the carbon fibers are well impregnated by the nanofilled resin and therefore part of the surrounding resin is retained even also after the strong etching procedure. From the micrographs in Fig. 10a-d at lager magnification, it is possible to observe that the single fibers are completely impregnated by resin layers.

This effect is also well observable in Fig. 10 showing a comparison between the single fiber of the fabric (without resin) (Fig. 10a) with the impregnated fibers (Fig. 10b-d).

The observation that the single fibers are well coated by the nanofilled resin, even after a strong etching procedure, highlights strong attractive interactions between the carbon fibers and the nanofilled resins. The carbon nanotubes embedded in the epoxy resin can be observed when high magnification, as those chosen for the micrographs of Fig. 11 and 12, are set up. Fig. 11 evidences the presence of some carbon nanotubes arranged preferentially through the section of the panel in the direction perpendicular to the plane. This particular morphological feature may be the key factor causing the high out of plane electrical conductivity. The reason why this peculiar morphology is obtained is most likely due to the adopted infiltration process where the nanofilled resin is forced to flow through the thickness of the preform.

Fig. 12 shows successively higher magnifications (from left to right) of the etched surface on the top side of the CFR panel surface in the region which has not been broken by the cutting. The images of Fig. 12 highlight a noteworthy result: the carbon nanotubes, dragged by the resin in which they are embedded, are able to pass through all the plies with no observable sieving. effect. This result is certainly due the non-usual technique to infuse the nano-filled resin into the carbon fiber dry preform.

Although the image of the final composite is different from the nanofilled resin of Fig. 5 (the composite is much more compact due to the presence of the carbon fibers), it is possible to observe CNTs bridging all the epoxy-rich regions between the plies, giving rise to an efficient network as for the nanofilled resin (without CFs). The presence of this network between CNTs
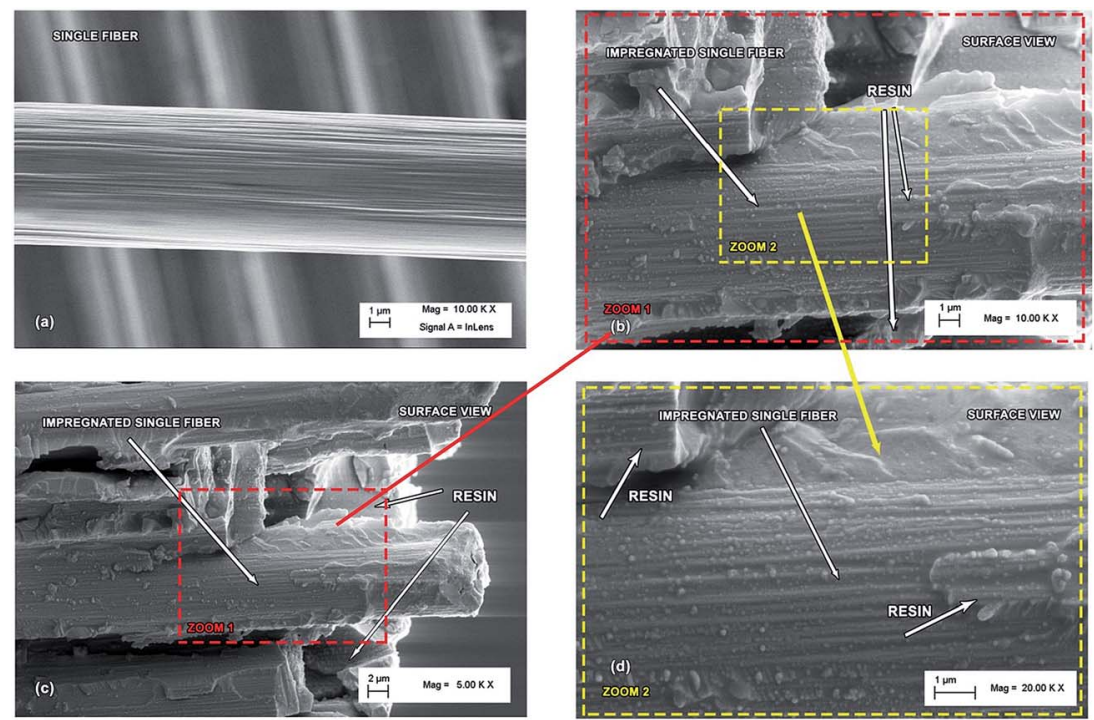

Fig. 10 SEM micrographs of single carbon fiber (a); detail at different magnification (b), (c) and (d) of a single CF impregnated by the nanofilled resin. 

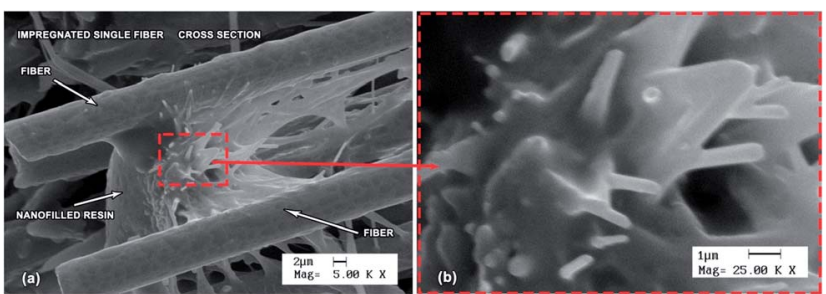

Fig. 11 SEM micrograph of carbon nanotubes in the resin impregnating the CFs and in the narrow matrix-rich regions between the fibers (a), detail of the presence of carbon nanotubes in the epoxy matrix (b).

is confirmed by the electrical measurements. In fact, the out of plane conductivity (across the sample thickness) reaches the value of $3.9 \mathrm{~S} \mathrm{~m}^{-1}$. This value is among the highest value reached until now for nanofilled resins impregnating carbon fibers of panels obtainable through the proposed simple manufacturing process which can be scaled up to industrial levels.

3.2.2. Electrical properties. The performance of the developed Carbon fiber/epoxy laminates composites as material able to be used in structural aeronautic application has been verified through the electrical characterization. Carbon fiber/epoxy laminate composites are heterogeneous materials and physical properties such as electrical conductivity depend on carbon fiber ply orientation. If no strategies are used to improve the transversal conductivity across the composite section, the thickness of the inter-laminar epoxy layer could be enough to electrically insulate the successive carbon fiber layer. Therefore, electrical performance of the adopted impregnating epoxy system and the effectiveness of the implemented manufacturing process was evaluated by measuring the electrical conductivity of the anisotropic manufactured panels. In particular, the in plane volume DC conductivity $\left(\sigma_{\mathrm{v} \|}\right)$ and the out of plane conductivity $\left(\sigma_{\mathrm{v} \perp}\right)$ have been determined and compared with data from literature (Table 4). In the observed range of temperature a $\sigma_{\mathrm{v} \|}$ going from $19.5 \mathrm{kS} \mathrm{m}^{-1}$ to $19.7 \mathrm{kS}$ $\mathrm{m}^{-1}$ is detected by performing measurements on the strip sample. A volume conductivity for the CFR panel of around
$20 \mathrm{kS} \mathrm{m}^{-1}$ is a typical value for aeronautic composite materials. ${ }^{24}$ Moreover as assumed in ${ }^{25,26}$ the in plane volume DC conductivity is equal to

$$
\sigma_{\mathrm{v} \|}=\sigma_{\mathrm{f}} V_{\mathrm{f}}
$$

where $\sigma_{\mathrm{f}}$ and $V_{\mathrm{f}}$ are the conductivity and volume fraction of the fiber, respectively. At room temperature $\sigma_{\mathrm{f}}=39 \mathrm{kS} \mathrm{m}^{-1}$ and this value is consistent with that reported in literature. ${ }^{25,27}$ The variation of $\sigma_{\mathrm{v} \|}$ with the temperature is in the range of the measurement accuracy $\left(\Delta \sigma_{\mathrm{v} \|}=2.8 \%\right.$ calculated by considering a confidence interval of $95 \%$, that correspond to $\pm 0.5 \mathrm{kS} \mathrm{m}^{-1}$ ), showing that temperature does not affect the electrical behaviour of the CFRC in the direction parallel to the fibers plane. These results are in good agreement with those by Keiji and Yoshihiro $^{28}$ who have found that the conductivity along the fiber direction (in angle) at $0^{\circ}$ and $45^{\circ}$ for the composites reinforced with continuous carbon fibers is almost independent of temperature. In the same range of temperature also the out of plane DC volume conductivity $\sigma_{\mathrm{v} \perp}$ shows a very small variation comparable with that associated to the measurement error. In fact, the conductivity assumes a value of $3.9 \mathrm{~S} \mathrm{~m}^{-1}$ at $T=30^{\circ} \mathrm{C}$ and a maximum of $4.1 \mathrm{~S} \mathrm{~m}^{-1}$ at $T=60{ }^{\circ} \mathrm{C}$, whereas the measurement accuracy of about $4 \%$ leads to a tolerance of less than $0.2 \mathrm{~S} \mathrm{~m}^{-1}$. Moreover, it is possible to observe that $\sigma_{\mathrm{v} \perp}$ is higher than the value found for the unfilled composite resin TG20BDCNTs, ${ }^{19}$ i.e. $0.03 \mathrm{~S} \mathrm{~m}^{-1}$ at $T=30^{\circ} \mathrm{C}$. This means that, as shown in Fig. 12, the CNTs are effective in establishing conductive paths along the fibers plane. As already evidenced, a $\sigma_{\mathrm{v} \perp}=3.9 \mathrm{~S} \mathrm{~m}^{-1}$ is among the highest values reached until now for very simple manufacturing processes. A value of the same order of magnitude has been reached only by adopting the electrophoresis technique. ${ }^{29}$ In fact, in the approach of ref. 29 , a first stage involving an electrophoresis process was carried out for the selective deposition of MWCNTs or single-walled carbon nanotubes (SWCNTs) on woven carbon fabric (Magnamite IM7 $-10 \mathrm{~cm} \times 15 \mathrm{~cm}$ ). The carbon fabric panels were subsequently infiltrated with epoxy resin using vacuum-assisted resin transfer molding (VARTM) to fabricate multiscale hybrid composites in which the nanotubes were completely integrated into the fiber

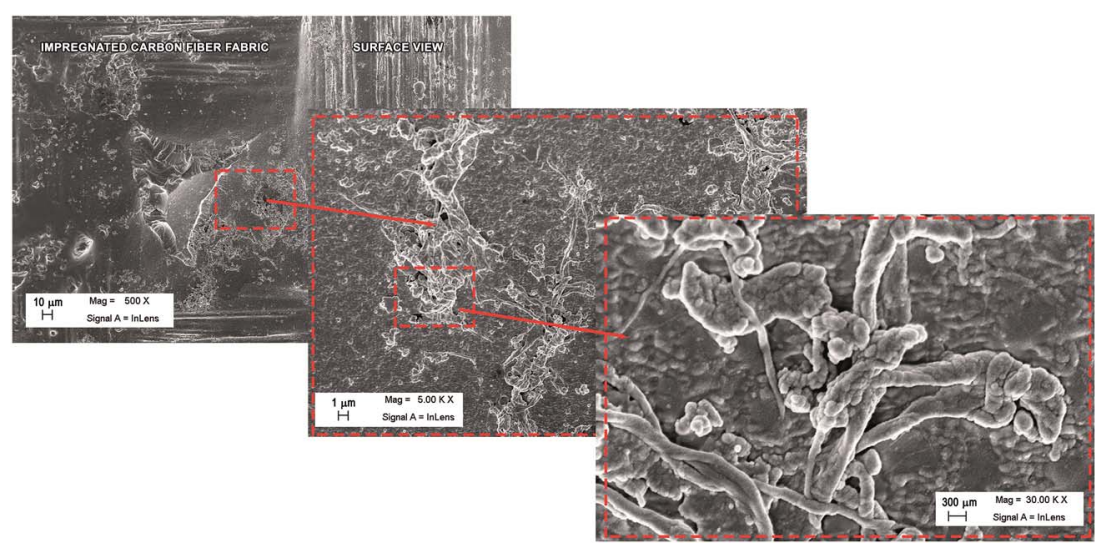

Fig. 12 SEM micrographs of the etched surface on the top side of the CFR panel surface in the region which has not been broken by the cutting. 
Table 4 Electrical conductivity values of the CFRC described in this paper (CFs impregnated with the nanofilled resin T20BDCNTs) and other CFRCs published in literature

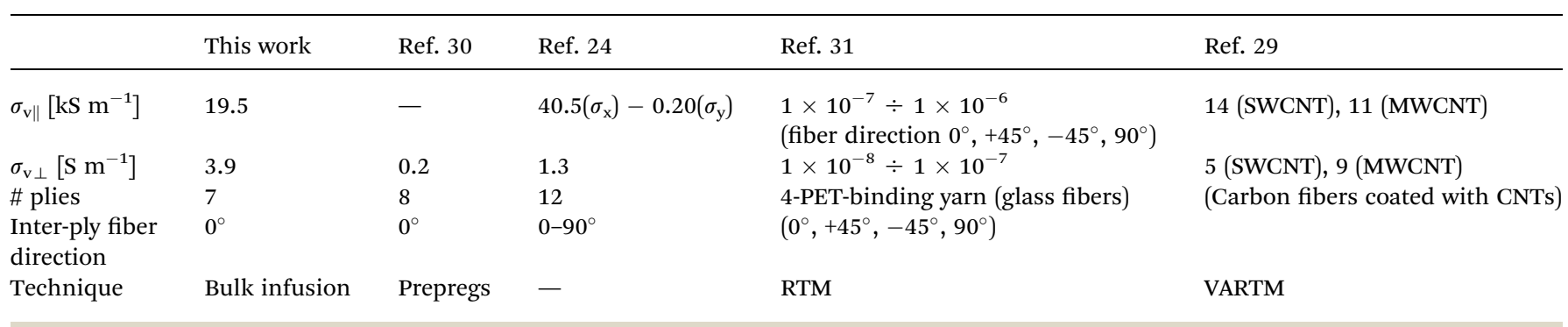

bundles. In our case, a processing technique more similar to those currently used by aircraft manufacturers was employed and no pre-treated carbon fibers coated with CNTs were employed. In Table 4, the results of interesting and careful experiments carried out in the last years for manufacturing appropriate CFRCs fulfilling the stringent requirements adopted for structural composites in aeronautic field are summarized.

In particular, the electrical conductivity values measured at room temperature for our panel (second column) are compared with those based on nanofilled epoxy resins already published in literature. In the case of ref. 31, Gojny et al. employs nanoparticle-reinforced FRP containing carbon black (CB) and CNTs epoxies as matrix for conventional glass fibre-reinforced panel (GFRP), manufactured via Resin Transfer Moulding process (RTM). The GFRP containing $0.3 \%$ wt of aminofunctionalized double-wall carbon nanotubes (DWCNT- $\mathrm{NH}_{2}$ ) were found to exhibit an anisotropic electrical conductivity, with the in plane conductivity of fiber fabric of one order of magnitude higher than the transversal conductivity. Piche et al. in ref. 24 presented different experimental approaches to characterize the electric behaviour of carbon fiber composites used in aeronautic industry and a numerical model to support test definition and material characterization. The validation of the models with experimental tests allowed to extract values of electrical conductivities of fiber reinforced composite samples, once the appropriate method to perform the test is selected. The developed approach on a composite having 12 plies with $0 / 90^{\circ}$ sequence of plies, allowed to obtain an out of plane volumetric conductivity of composites $\sigma_{\mathrm{v} \perp}=1.3 \mathrm{~S} \mathrm{~m}^{-1}$, and in plane values of $\sigma_{\mathrm{x} \|}=40.5 \mathrm{kS} \mathrm{m}^{-1}$ and $\sigma_{\mathrm{y} \|}=0.20 \mathrm{kS} \mathrm{m}^{-1}$. In our case (see column 2 in Table 4), the type of used fabric and the chosen layup direction allows to obtain an in plane value independent on the axis. Lonjon A. et al. ${ }^{30}$ obtained an electrical conductivity improvement of aeronautical carbon fiber reinforced polyepoxy composites by CNTs inclusion. Carbon fiber composite were prepared by prepregs, with epoxy multicomponent resin alone and with epoxy multicomponent resin filled with a small CNT weight fraction. Palmitic acid was used as dispersing agent of CNTs in epoxy systems, which enables the realization of the percolation threshold at low rates (about $0.4 \%$ wt of CNTs). For CF composites, 8 prepregs were assembled with unidirectional carbon fiber oriented in the same direction $\left(0^{\circ}\right)$. Electrical measurements of the volumetric conductivity of unfilled and filled were performed in the frequency domain $\left[10^{-2} ; 10^{6}\right] \mathrm{Hz}$, at room temperature. The results highlights a poor conductivity value $\left(\sigma_{\mathrm{v} \perp} \cong 7.1 \times 10^{-3} \mathrm{~S} \mathrm{~m}^{-1}\right)$ in the out of plane measurement for the laminate obtained with unfilled resin. The addition of CNTs in the epoxy resin, causes a large increase in the conductivity of the laminate composites $\left(\sigma_{\mathrm{v} \perp} \cong 0.2 \mathrm{~S} \mathrm{~m}^{-1}\right)$. Summing up, by considering the values shown in Table 4 , we can conclude that the values exhibited by our panels for the out of plane conductivity are among the highest values obtained up to now with the exception of ref. 29, where higher values were achieved using a more complex manufacturing process. Although it is very hard to directly obtain conclusions from the comparison between the different data above discussed and shown in Table 4, because of the relevant differences in the epoxy chemical composition, nanofiller nature, concentration and manufacturing process, a comparison is the only potential way to address technological solutions towards the required targets in aeronautic field. Experiments are in progress to better understand the effect of the nature of the components and process manufacturing on the electrical properties.

\section{Conclusions}

In this paper we have shown the first results obtained using a non-usual technique to manufacture CFRCs. This technique is particularly advantageous to impregnate CFs with aeronautic resins filled with conductive nanofillers. In particular, the impregnation can be obtained also using an epoxy mixture characterized by viscosity values higher than $0.3 \mathrm{~Pa}$ s.

The anisotropic volumetric DC conductivity of the CFRC is almost independent on temperature in the range $\left[30 ; 90{ }^{\circ} \mathrm{C}\right.$ and is about $20 \mathrm{kS} \mathrm{m}^{-1}$ for the in plane value and $3.9 \mathrm{~S} \mathrm{~m}^{-1}$ for the out of plane at $T=30^{\circ} \mathrm{C}$. This last value is among the highest value found for CFRCs impregnated with resins loaded with carbon nanotubes and manufactured by means of very simple processes.

Moreover, the in plane value confirm that the conduction mechanisms is governed by the fibers. In fact, the obtained volumetric conductivity of the strip sample is close to that of carbon fibers alone whose conductivity is around $10^{4}$ to $10^{5} \mathrm{~S}$ $\mathrm{m}^{-1}$. The value achieved for the out of plane conductivity which is almost one order of degree higher than that of the used nanofilled resin alone, shows that the electrical conduction is 
improved by the contribution of the percolating paths created by the MWCNTs inside the resin.

\section{Acknowledgements}

The research leading to these results has received funding from the European Union's Seventh Framework Programme for research, technological development and demonstration under Grant Agreement no. 313978.

\section{References}

$1 \mathrm{~J}$. K. Fink, Reactive Polymers Fundamentals and Applications: A Concise Guide to Industrial Polymers, Plastics Design Library (PDL), William Andrew, Norwich, NY, U.S.A, 2005, ISBN: 0-8155-1515-4.

2 C. A. May, Epoxy Resins Chemistry and Technology, Second edn, Revised and Expanded, ed. C. A. May, Marcel Dekker, Inc, New York, 1988, ISBN: 0-8247-7690-9.

3 L. Guadagno, L. Vertuccio, A. Sorrentino, M. Raimondo, C. Naddeo, V. Vittoria, G. Iannuzzo, E. Calvi and S. Russo, Mechanical and barrier properties of epoxy resin filled with multi-walled carbon nanotubes, Carbon, 2009, 47, 24192430.

4 J. K. W. Sandler, J. E. Kirk, I. A. Kinloch, M. S. P. Shaffer and A. H. Windle, Ultra-low electrical percolation threshold in carbon-nanotube-epoxy composites, Polymer, 2003, 44, 5893-5899.

5 R. Khare and S. Bose, Carbon nanotube based composites - A review, J. Miner. Mater. Charact. Eng., 2005, 4, 31-46.

6 L. Guadagno, B. De Vivo, A. Di Bartolomeo, P. Lamberti, A. Sorrentino, V. Tucci, L. Vertuccio and V. Vittoria, Effect of functionalization on the thermo-mechanical and electrical behavior of multi-wall carbon nanotube/epoxy composites, Carbon, 2011, 49, 1919-1930.

7 N. Hu, Z. Masuda, C. Yan, G. Yamamoto, H. Fukunaga and T. Hashida, The electrical properties of polymer nanocomposites with carbon nanotube fillers, Nanotechnology, 2008, 19, 215701-215711.

8 D. S. Mclachlan, C. Chiteme, C. Park, K. E. Wise, S. E. Lowther, P. T. Lillehei, E. J. Siochi and J. S. Harrison, $\mathrm{AC}$ and DC percolative conductivity of single wall carbon nanotube polymer composites, J. Polym. Sci., Part B: Polym. Phys., 2005, 43, 3273-3287.

9 L. Guadagno, M. Raimondo, V. Vittoria, L. Vertuccio, K. Lafdi, B. De Vivo, P. Lamberti, G. Spinelli and V. Tucci, The role of carbon nanofiber defects on the electrical and mechanical properties of CNF-based resins, Nanotechnology, 2013, 24, 305704(10).

10 B. E. Kilbride, J. N. Coleman, J. Fraysse, P. Fournet, M. Cadek, A. Drury, S. Hutzler, S. Roth and W. J. Blau, Experimental observation of scaling laws for alternating current and direct current conductivity in polymer-carbon nanotube composite thin films, J. Appl. Phys., 2002, 92, 4024-4030.

11 L. Guadagno, C. Naddeo, V. Vittoria, A. Sorrentino, L. Vertuccio, M. Raimondo, V. Tucci, B. De Vivo,
P. Lamberti, G. Iannuzzo, E. Calvi and S. Russo, Cure behavior and physical properties of epoxy resin-filled with multiwalled carbon nanotubes, J. Nanosci. Nanotechnol., 2010, 10, 2686-2693.

12 B. De Vivo, P. Lamberti, V. Tucci, L. Guadagno, L. Vertuccio, V. Vittoria and A. Sorrentino, Comparison of the physical properties of epoxy-based composites filled with different types of carbon nanotubes for aeronautic applications, Adv. Polym. Technol., 2012, 31, 205-218.

13 L. Guadagno, M. Raimondo, V. Vittoria, L. Vertuccio, C. Naddeo, P. Lamberti and V. Tucci, Italian Patent Resina epossidica con basso tenore di umidità no. TO2013A000926, 15 November 2013.

14 L. Guadagno, M. Raimondo, P. Longo, L. Bonnaud, O. Murariu and P. Dubois, Italian Patent Resina epossidica multifunzionale con accresciuta resistenza alla fiamma no. TO2013A001021, 13 December 2013.

15 L. Guadagno, M. Raimondo, K. Lafdi, A. Fierro, S. Rosolia and M. R. Nobile, Influence of Nanofiller Morphology on the Viscoelastic Properties of CNF/Epoxy Resins, AIP Conf. Proc., 2014, 1599, 386-389.

16 M. R. N. Nobile, M. Raimondo, K. Lafdi, A. Fierro, S. Rosolia and L. Guadagno, Relationships between Nanofiller Morphology and Viscoelastic Properties in CNF/Epoxy Resins, Polym. Compos., 2015, (accepted for publication).

17 L. Guadagno, M. Raimondo, V. Vittoria, L. Vertuccio, C. Naddeo, S. Russo, B. De Vivo, P. Lamberti, G. Spinelli and V. Tucci, Development of epoxy mixtures for application in aeronautics and aerospace, RSC Adv., 2014, 4, 15474-15488.

18 M. Raimondo, S. Russo, L. Guadagno, P. Longo, S. Chirico, A. Mariconda, L. Bonnaud, O. Murariu and Ph. Dubois, Effect of Incorporation of POSS compounds and phosphorous hardeners on Thermal and Fire Resistance of nanofilled Aeronautic Resins, RSC Adv., (in revision)

19 B. De Vivo, L. Guadagno, P. Lamberti, M. Raimondo, G. Spinelli, V. Tucci, L. Vertuccio and V. Vittoria, Electrical properties of multi-walled carbon nanotube/tetrafunctional epoxy-amine composites, AIP Conf. Proc., 2012, 1459, 199201.

20 M. Raimondo and L. Guadagno, Effect of incorporation of carbon nanotubes on the mechanical properties of epoxyamine composites, AIP Conf. Proc., 2012, 1459, 226-228.

21 F. Hussain, M. Hojjati, M. Okamoto and R. E. Gorga, Review article: Polymer-matrix Nanocomposites, Processing, Manufacturing, and Application: An Overview, J. Compos. Mater., 2006, 40, 1511-1575.

22 J. Summerscales and T. J. Searle, Low Pressure (Vacuum Infusion) Techniques for Moulding Large Composite Structures, Proc. Inst. Mech. Eng., Part L, 2005, 219, 45-58, DOI: $10.1243 / 146442005 X 10238$.

23 D. D. L. Chun, Carbon Fiber Composites, ed. D. L. Chun Deborah, Butterworth-Heinemann, 313 Washington Street Newton, MA 02158, 1994, ISBN: 0-7506-9169-7.

24 A. Piche, I. Revel and G. Peres, Experimental and Numerical Methods to Characterize Electrical Behaviour of Carbon Fiber Composites Used in Aeronautic Industry, in Advances 
in Composite Materials - Analysis of Natural and Man-Made Materials, ed. Pavla Tesinova, Publisher InTech, Rijeka, Croatia, 2011, ch. 20, pp. 481-496, ISBN 978-953-307-449-8.

25 N. Athanasopoulos and V. Kostopoulos, Prediction and experimental validation of the electrical conductivity of dry carbon fiber unidirectional layers, Composites, Part B, 2011, 42, 1578-1587.

26 M. Weber and M. R. Kamal, Estimation of the volume resistivity of electrically conductive composites, Polym. Compos., 1997, 18, 711-725.

27 V. Šafářová and J. Grég, Electrical conductivity measurement of fibers and yarns, 7th International Conference, TEXSCI, Liberec, Czech Republic, September 6-8, 2010.

28 O. Keiji and T. Yoshihiro, Electrical properties of carbon fiber reinforced plastics and their application, J. Jpn. Soc. Compos. Mater., 2002, 28, 228-234.
29 E. Bekyarova, E. T. Thostenson, A. Yu, H. Kim, J. Gao, J. Tang, H. T. Hahn, T. W. Chou, M. E. Itkis and R. C. Haddon, Multiscale Carbon Nanotube-Carbon Fiber Reinforcement for Advanced Epoxy Composites, Langmuir, 2007, 23, 39703974.

30 A. Lonjon, P. Demont, E. Dantras and C. Lacabanne, Electrical conductivity improvement of aeronautical carbon fiber reinforced polyepoxy composites by insertion of carbon nanotubes, J. Non-Cryst. Solids, 2012, 358, 1859-1862.

31 F. H. Gojny, M. H. G. Wichmann, B. Fiedler, W. Bauhofer and K. Schulte, Influence of nano-modification on the mechanical and electrical properties of conventional fibrereinforced composites, Composites, Part A, 2005, 36, 15251535. 